Article

\title{
Spacetime Topology and the Laws of Black Hole-Soliton Mechanics
}

\author{
Hari K. Kunduri \\ Department of Mathematics and Statistics, Memorial University of Newfoundland, \\ St. John's, NL A1C 2S6, Canada; hkkunduri@mun.ca; Tel.: +1-709-864-4358 \\ Academic Editor: David Kubiznak \\ Received: 28 October 2016; Accepted: 12 January 2017; Published: 17 January 2017
}

\begin{abstract}
The domain of outer communication of an asymptotically flat spactime must be simply connected. In five dimensions, this still allows for the possibility of an arbitrary number of 2-cycles supported by magnetic flux carried by Maxwell fields. As a result, stationary, asymptotically flat, horizonless solutions-"gravitational solitons"-may exist with non-vanishing mass, charge, and angular momenta. These gravitational solutions satisfy a Smarr-like relation, as well as a first law of mechanics. Furthermore, the presence of solitons leads to new terms in the well-known first law of black hole mechanics for spacetimes containing black hole horizons and non-trivial topology in the exterior region. I outline the derivation of these results and consider an explicit example in five-dimensional supergravity.
\end{abstract}

Keywords: black holes in higher dimensions; supergravity; black hole thermodynamics; gravitational aspects of string theory

\section{Introduction}

The proof of the black hole uniqueness theorem is a central achievement in general relativity. This result asserts that the any stationary, asymptotically flat analytic solution of the Einstein-Maxwell equations describing a black hole spacetime must belong to the three-parameter Kerr-Newman family of solutions (see the review [1]). There are a number of profound consequences of this theorem. In particular, it implies that if one starts with a black hole with mass $M$, angular momentum $J$ and electric charge $Q$ and considers an infinitesimal variation in the phase space of black holes, one must arrive at another member of the Kerr-Newman solution with parameters $M+\delta M, J+\delta J$ and $Q+\delta Q$. From the explicit form of he Kerr-Newman solution it is simple to derive the well-known first law of black hole mechanics

$$
\delta M=\frac{\kappa \delta A_{H}}{8 \pi}+\Omega_{H} \delta J+\Phi_{H} \delta Q
$$

where $A_{H}$ is the area of a spatial cross-section of the event horizon and $\kappa, \Omega_{H}$ and $\Phi_{H}$ are the surface gravity, angular velocity and electric potential of the horizon respectively. The above equation can be interpreted as an equation for the tangent space at a given point in the phase space; it forms an important part in the interpretation of a black hole as a thermodynamic system. Note that (1) was proved independently of the black hole uniqueness results [2]; it follows directly from an analysis of Einstein's equations for stationary, axisymmetric linearized perturbations.

The study of black hole solutions in spacetime dimension $D>4$ has been an active field of research the past two decades, chiefly motivated by developments in string theory and the gauge theory-gravity correspondence [3]. This is also a problem of intrinsic interest in the mathematical study of Einstein's equations, helping to illuminate features unique to $D=4$ [4]. We will focus here on asymptotically flat solutions in $D=5$ as it is the next simplest case, and arises naturally in the context of intersecting D-brane systems in string theory. In addition to the Myers-Perry vacuum 
solution [5], the natural generalization of the Kerr solution, which has horizon topology $H \simeq S^{3}$, a striking result was the discovery by Emparan and Reall of a vacuum black hole ('black ring') with $H \simeq S^{1} \times S^{2}$ [6]. Both families are characterized by a mass $M$ and two independent angular momenta $\left(J_{1}, J_{2}\right)$. Remarkably, it can be shown that the uniqueness theorems fail: a black hole in not characterized by its mass and angular momenta. Very recently, the author and Lucietti have constructed a family of 'black lens' solutions $\left(H \simeq S^{3} / \mathbb{Z}_{2}\right)[7,8]$. These three cases exhaust all allowed horizon topologies within the symmetry class of $U(1)^{2} \equiv U(1) \times U(1)$-invariant solutions [9].

It is natural to expect that the first law of mechanics in $D=5$ should take a more complicated form than (1) because there are more "directions" in the phase space of solutions. This problem has only recently been analyzed in generality [10] and the purpose of this article is to discuss and sharply summarize the salient results and provide some new results. In particular, we derive new formulae for the charge (27) and angular momenta (25) as well as associated potentials. First, however, we should discuss another class of solutions to the Einstein-Maxwell equations, which we refer to as gravitational solitons. These are stationary, asymptotically flat (we do not consider asymptotically locally Euclidean or asymptotically locally flat spacetimes, e.g., the Eguchi-Hanson or Euclidean Schwarzschild metrics with an extra timelike direction $-\mathrm{d} t^{2}$ added for which the standard ADM mass is not defined. For further details on this issue, see [11]) horizonless spacetimes with non-zero energy. Such spacetimes do not in fact exist in $D=4-$ a result that can be succinctly summarized by the slogan "no solitons without horizons" [12]. This conclusion is closely related to the fact that in $D=4$, the region exterior to a black hole horizon (the domain of outer communication) must be simply connected [13]. Roughly, this implies a spacetime without a horizon must be topologically trivial and does not carry any non-zero conserved charges (although if there are non-Abelian Yang-Mills fields present, topology can be contained within non-trivial gauge field configurations [14]). In $D=5$ the restriction of simple connectedness is weaker, and indeed a horizonless spacetime could contain an arbitrary number of 2-cycles ("bubbles"). More precisely, if $\Sigma$ is a spatial hypersurface in the domain of outer communication, then the second homology $\mathrm{H}_{2}(\Sigma)$ can contain non-trivial elements. As we describe below, these 2-cycles may carry magnetic flux sourced by Maxwell fields and and contribute towards a non-zero mass, angular momenta, and electric charge of the spacetime.

These solitons (variously known as "smooth geometries" or "fuzzballs" in the literature, see e.g., [15] and references therein) appear to play an important role in the lack of black hole uniqueness in $D=5$. A study of this assertion has been initiated in [16]. Here we will be concerned with the problem of proving an analogue of (1) valid for gravitational solitons, which we will refer to as the first law of soliton mechanics. Further, we will consider the possibility of black hole solutions containing solitons in their domain of outer communication. This will lead to a modification of (1) to include the effects of the 2-cycles, producing a fist law of "black hole and soliton mechanics" [10]. The consequences of these new terms of black hole thermodynamics is only starting to be explored.

\section{Black Holes and Solitons in Five Dimensions}

We begin by outlining the framework for analyzing black hole and soliton solutions $(\mathcal{M}, g)$ in five-dimensional gravity coupled to an arbitrary number of Maxwell fields $F^{I}, I=1 \ldots N$ and neutral scalar fields $\chi^{A}, A=1 \ldots n$. We refer to the reader to the review articles [3,4] for a comprehensive discussion on known solutions in $D \geq 5$ and the statements of the strongest current uniqueness and rigidity results.

\subsection{Stationary, Biaxisymmetric Solutions}

We will consider spacetime solutions $\left(\mathcal{M}, g, F^{I}, \chi^{A}\right)$ that are stationary and biaxisymmetric. This means that the spacetime manifold admits an action of $\mathbb{R}_{t} \times U(1)^{2}$ as isometries. In addition we require that the isometry group also extend to symmetries of the matter fields. We denote the 
generator of the timelike isometry that coincides the asymptotic generator of time translations by $\xi$ and the generators of the rotational symmetries $m_{i}$. The invariance properties above can be expressed as

$$
\mathcal{L}_{\xi_{\alpha}} g=\mathcal{L}_{\xi_{\alpha}} F^{I}=\mathcal{L}_{\xi_{\alpha}} \chi^{A}=0
$$

where $\xi_{\alpha}=\left(\xi, m_{i}\right)$ and $\mathcal{L}$ denotes Lie differentiation. If the spacetime contains a black hole, we will take $\xi$ to be stationary Killing field which becomes null on the event horizon.

Due to (2) it proves convenient to consider all spacetime fields as functions on the two-dimensional orbit space $\mathcal{B} \equiv \mathcal{M} /\left(\mathbb{R}_{t} \times U(1)^{2}\right) . \mathcal{B}$ can be shown to be a simply connected manifold with boundary $\partial \mathcal{B}$ and corners [9]. In the interior, on the $1 \mathrm{~d}$ boundary segments (except the part corresponding to the event horizon in the spacetime) and at the corners (where these segments intersect), the matrix of the scalar products of Killing fields $g\left(m_{i}, m_{j}\right)$ has rank 2,1,0 respectively. For an asymptotically flat spacetime, the orbit space $\mathcal{B}$ is non-compact with one asymptotic end corresponding to spatial infinity. The boundary $\partial \mathcal{B} \cong \mathbb{R}$ is divided into intervals $I$, or "rods", all of which are compact except for the two asymptotic ends. If a black hole is present, the horizon, which we assume to be connected, corresponds to a compact interval $I_{H} \cong H / U(1)^{2}$. The rest of $\partial \mathcal{B}$ correspond to 2-surfaces in the spacetime where some integer linear combination $v^{i} m_{i}, v^{i} \in \mathbb{Z}$ of the Killing fields $m_{i}$ vanish. These 2-surfaces are in fact topologically spheres, where the endpoints of a finite rod correspond to the "poles" of the $S^{2}$ where the same spatial Killing field has fixed points. The two semi-infinite intervals, which we denote by $I_{+}, I_{-}$, correspond to the axes of rotation in the spacetime which extend out to spatial infinity.

The specification of the rod data (i.e., length and location of the rods $I_{a}, I_{H}$ along with the associated integers $\left.v_{a}^{i}\right)$ fully characterize a $\mathbb{R} \times U(1)^{2}$-invariant spacetime $(\mathcal{M}, g)$ up to diffeomorphism [9]. Indeed, the appropriate generalization of the $4 \mathrm{~d}$ uniqueness theorems state that an asymptotically flat $\mathbb{R} \times U(1)^{2}$-invariant black hole solution of the vacuum equations is uniquely specified by its mass $M$, angular momenta $J_{i}$, and rod data [9]. The latter fixes the horizon topology $H$ of the black hole, as well as the topology of a spatial hypersurface $\Sigma$ in the domain of outer communication. In the following sections, we will exploit this framework in order to reduce various Komar-type integrals defined on $\Sigma$ to integrals over $\partial \mathcal{B}$. This will lead to a generalization of the variational law (1).

\subsection{The Theory and Potentials}

Our primary interest is in the five-dimensional supergravity theories that govern the low-energy dynamics on appropriately compactified string theory. For the sake of generality we will consider the theory with action

$$
S=\frac{1}{16 \pi} \int \star R-f_{A B}(\chi) \mathrm{d} \chi^{A} \wedge \star \mathrm{d} \chi^{B}-g_{I J}(\chi) F^{I} \wedge \star F^{J}-\frac{1}{6} C_{I J K} F^{I} \wedge F^{J} \wedge A^{K}
$$

where $F^{I}=\mathrm{d} A^{I}$ for a locally defined gauge potential $A^{I}$, the couplings $f_{A B}, g_{I J}$ are positive definite and $C_{I J K}=C_{(I J K)}$ are constants. One may also include a scalar potential term $V(\chi) \star 1$ to the action, which we will not do for simplicity (see [10]). The action (3) includes pure Einstein-Maxwell theory and minimal supergravity coupled to $N$ vector multiplets, which arises from Type IIB supergravity on $K_{3}$ or $S^{1} \times T^{4}$. Note that it does not include gauged supergravities which are relevant in the context of the gauge theory-gravity correspondence; our analysis does not apply to asymptotically Anti-de Sitter spacetimes.

As observed by GIbbons and Warner [17], to include the effects of 2-cycles, it is important not to assume that the gauge fields are globally defined-indeed it is precisely the existence of non-trivial elements of $H^{2}(\Sigma)$ that physically "support" bubbles from collapsing. To avoid this we work dual Maxwell fields $G^{I} \equiv \star F^{I}$. The field equations following from (3) are

$$
R_{a b}=g_{I J}\left(\frac{2}{3} F_{a c}^{I} F_{b}^{J c}+\frac{1}{6} G_{a c d}^{I} G_{b}^{J c d}\right)+f_{A B} \partial_{a} \chi^{A} \partial_{b} \chi^{B}
$$


and

$$
\nabla^{b}\left(g_{I J} F_{b a}^{J}\right)+\frac{1}{8} C_{I J K} F^{J b c} G_{a b c}^{K}=0 .
$$

with an additional set of equations for the scalar fields $\chi^{A}$.

We define the mass, angular momenta, and charge contained in the asymptotically flat spacetime by the integrals over the "boundary" sphere $S_{\infty} \equiv \lim _{r \rightarrow 0} S_{r}$ where $S_{r}$ is a sphere of radius $r$ in the Cartesian coordinates defined at spatial infinity:

$$
M=-\frac{3}{32 \pi} \int_{S_{\infty}} \star \mathrm{d} K, \quad J_{i}=\frac{1}{16 \pi} \int_{S_{\infty}} \star \mathrm{d} m_{i}, \quad Q_{I} \equiv \frac{1}{8 \pi} \int_{S_{\infty}} g_{I J} \star F^{J}
$$

where $K$ is the asymptotic generator of timelike translations (if the spacetime contains a black hole, $K=\xi-\Omega_{i} m_{i}$, and otherwise it coincides with $\left.\xi\right)$. We will refer to the quantities (6) as conserved charges.

We now show how the symmetries (2) and the field Equations (4) and (5), lead to existence of potentials globally defined on $\mathcal{B}$. Closure of the $F^{I}$ imply the existence of potentials $\Phi^{I}$ defined by

$$
\mathrm{d} \Phi^{I}=i_{\xi} F^{I}
$$

and since $\mathcal{B}$ is simply connected, $\Phi^{I}$ are globally defined. The integration constant is fixed by requiring that $\Phi^{I}$ vanish at spatial infinity. Invariance of $\star G^{I}$ implies the existence of globally defined closed, but necessarily exact 2 -forms

$$
\Theta_{I} \equiv g_{I J} i_{\xi} G^{J}-\frac{1}{2} C_{I J K} F^{J} \Phi^{K}
$$

Now, applying Stokes' theorem to the mass integral and applying the Killing field identity $\star \mathrm{d} \star \mathrm{d} \xi=-2 R(\xi)$ where $R(\xi)$ is the one-form obtained by contracting the Ricci tensor (4) with $\xi$, one finds [10]

$$
M=\frac{3 \kappa A_{H}}{16 \pi}+\frac{3}{2} \Omega_{i} J_{i}+\frac{1}{16 \pi} \int_{\Sigma} \Theta_{I} \wedge F^{I}+\frac{1}{8 \pi} \int_{H} \Phi^{I} g_{I J} \star F^{J}
$$

Here $\Sigma$ is a spatial hypersurface with asymptotic boundary $S_{\infty}^{3}$ and, if a black hole is present, an "inner" boundary component $-H$. In the absence of a horizon, the first two terms in (9) are not present. The important term is the integral involving $\Theta_{I}$; this would vanish if $\Theta_{I}$ is an exact form, but if there are 2-cycles present (e.g., for a soliton spacetime) this term will contribute to the mass of the spacetime.

The rotational symmetries may now be used to derive the existence of closed forms and globally defined potentials as follows. We have

$$
i_{m_{i}} F^{I}=\mathrm{d} \Phi_{i}^{I}
$$

which define globally defined "magnetic" potentials. Further the invariance property $\mathcal{L}_{m_{i}} \Theta_{I}=0$ leads to globally defined scalar potentials $U_{I i}$ defined by

$$
\mathrm{d} U_{I i}=i_{m_{i}} \Theta_{I}+\frac{1}{2} C_{I J K} \mathrm{~d} \Phi_{i}^{J} \Phi_{H}^{K}
$$

Similarly there are globally defined closed two-forms given by

$$
Y_{I}^{i} \equiv g_{I J} i_{m_{i}} G^{J}-\frac{1}{2} C_{I J K} F^{J} \Phi_{i}^{K}
$$

From Cartan's formula we obtain globally defined scalar fields $\mu_{i j}^{I}$ defined by $\mathrm{d} \mu_{i j}^{I}=i_{m_{i}} \mathrm{Y}_{j}^{I}$. We will demonstrate below how the potentials $\left(\Phi_{i}^{I}, U_{I i}, \mu_{I i}\right)$ can be used to express the mass, angular momenta, and charge of the spacetime in terms of contributions from 2-cycles. 


\section{1st Law of Black Hole-Soliton Mechanics}

We now consider variations $\left(\delta g, \delta F^{I}, \delta \chi^{A}\right)$ about a given solution $\left(g, F^{I}, \chi^{A}\right)$. We assume the variations satisfy the linearized equations of motion following from (4), (5) and that are invariant under the $\mathbb{R} \times U(1)^{2}$ symmetry. There are additional assumptions that must be made (e.g., the Killing fields are the same, so $\delta \xi=\delta m_{i}=0$ and the location of the event horizon $H$ and any 2-cycles is unchanged). These conditions are standard in the classic proof of the first law of black hole mechanics [2]. Starting from the ADM mass formula for the perturbation

$$
\delta M=\frac{3}{8 \pi} \int_{S_{\infty}} \star(K \wedge h)
$$

where $h_{a b}=\frac{1}{2}\left(\nabla^{b} \delta g_{b a}-g^{c d} \nabla_{a} \delta g_{c d}\right)$, an involved calculation using the linearized equations of motion and another application of Stokes' theorem leads to the mass variation formula [10]

$$
\delta M=\frac{\kappa \delta A_{H}}{8 \pi}+\Omega_{i} \delta J_{i}+\frac{1}{8 \pi} \int_{\Sigma} \Theta_{I} \wedge \delta F^{I}+\frac{1}{8 \pi} \int_{H} \Phi^{I} \delta\left(g_{I J} \star F^{J}\right)
$$

Our task it to express $\delta M$ in terms of variations of the potentials and charges defined above. We will use the identity

$$
\int_{\Sigma} \omega=2 \pi^{2} \int_{\mathcal{B}} \eta_{i j} i_{m_{j}} i_{m_{i}} \omega
$$

for any 4 -form $\omega$ where $\eta_{i j}$ is the antisymmetric symbol with $\eta_{12}=1$ and $m_{i}$ have $2 \pi$-periodic orbits.

\subsection{Soliton Spacetimes}

First consider the case where spacetime does not contain any horizons, so $\Sigma$ is complete with an asymptotically flat end. The orbit space consists of two semi-infinite intervals $I_{ \pm}$upon which a certain linear combination of Killing fields vanish (say $m_{1}$ and $m_{2}$ respectively) and finite-length rods $I_{a}$ upon which the Killing field $v_{a}^{i} m_{i}$ vanishes. The latter correspond to $S^{2}$-submanifolds. From their definition it is easy to see that the associated potentials $v_{a}^{i} \Phi_{i}^{I}, v_{a}^{i} U_{I i}, v_{a}^{i} \mu_{j i}^{I}$ must be constant on $I_{a}$. In particular on $I_{ \pm}$, the associated potentials vanish, since they are constants that vanish at spatial infinity.

The Smarr relation for the mass (9) in the soliton case is simply

$$
M=\frac{1}{16 \pi} \int_{\Sigma} \Theta_{I} \wedge F^{I}=\frac{\pi}{4} \int_{\mathcal{B}} \eta_{i j} \mathrm{~d} U_{I j} \wedge \mathrm{d} \Phi_{i}^{I}
$$

where we have used (15). The integral can be simplified by using Stokes' theorem on the manifold-with-boundary $\mathcal{B}$ :

$$
\int_{\mathcal{B}} \eta_{i j} \mathrm{~d} U_{I j} \wedge \mathrm{d} \Phi_{i}^{I}=\int_{\partial \mathcal{B}} \eta_{i j} U_{I j} \mathrm{~d} \Phi_{i}^{I}
$$

where we used vanishing of $U_{I i}$ at spatial infinity to eliminate the integral over the asymptotic end $\mathcal{B}_{\infty}$ corresponding to the asymptotically flat in the spacetime. Furthermore there are no contributions from $I_{ \pm}$either (note that either $U_{I+}=\Phi_{+}=0$ on $I_{+}$and similarly on $I_{-}$). We are left with an integral over the remaining finite rods. Suppose we are on a interval on which $v_{a}^{i} m_{i}=0$. By an $S L(2, \mathbb{Z})$ transformation we can work in a basis in which $\hat{m}_{1}=v_{a}^{i} m_{i}$ and $\hat{m}_{2}=w_{i} m_{i}$ where $w_{i} \in \mathbb{Z}$. Here $\hat{m}_{2}$ represents the other Killing field which does not vanish in the interior of $I_{a}$ but does vanish at its endpoints. Thus this describes a 2-cycle, i.e., a non-trivial element of $H_{2}(\Sigma)$. The transformation preserves the periodicity of the orbits of $\hat{m}_{i}$. Working in this basis, and noting $\left(\hat{U}_{I 1}, \hat{\Phi}_{1}^{I}\right)$ are constant on a given rod $I$,

$$
\int_{I} \eta_{i j} U_{I j} \mathrm{~d} \Phi_{i}^{I}=\int_{I} \eta_{i j} \hat{U}_{I j} \mathrm{~d} \hat{\Phi}_{i}^{I}=-\hat{U}_{I 1} \int_{I} \mathrm{~d} \hat{\Phi}_{2}^{I}=\frac{2}{\pi} \Psi_{I}[C] q^{I}[C]
$$


where we have defined the magnetic flux

$$
q^{I}[C] \equiv \frac{1}{4 \pi} \int_{C} F^{I}
$$

and the potentials $\Psi_{I}[C] \equiv \pi v^{i} U_{I i}=\pi \hat{U}_{I i}$. A similar argument applied to the mass variation formula (14) without horizon terms gives

$$
\frac{1}{8 \pi} \int_{\Sigma} \Theta_{I} \wedge \delta F^{I}=\int_{\mathcal{B}} \eta_{i j} \mathrm{~d} U_{I j} \wedge \mathrm{d} \delta \Phi_{i}^{I}=\Psi_{I}[C] \delta q^{I}[C]
$$

Therefore, for spacetimes containing gravitational solitons without horizons we arrive at

$$
\begin{aligned}
M & =\frac{1}{2} \sum_{[C]} \Psi_{I}[C] q^{I}[C], \\
\delta M & =\sum_{[C]} \Psi_{I}[C] \delta q^{I}[C],
\end{aligned}
$$

which we refer to as the mass formula and first law of soliton mechanics respectively [10]. Of course in the absence of a horizon or any 'temperature' the possibility of a thermodynamic interpretation is unclear.

Let us now turn to finding an analogous expression to (21) for $J_{i}=J\left[m_{i}\right]$. Using Stokes' theorem,

$$
J\left[m_{k}\right]=\frac{1}{8 \pi} \int_{\Sigma} \star R\left(m_{k}\right)=\frac{1}{8 \pi} \int_{\Sigma} g_{I J}\left(\frac{2}{3} i_{m_{k}} F^{I} \wedge G^{J}-\frac{1}{3} i_{m_{k}} G^{I} \wedge F^{J}\right)
$$

where we have used (4). Using (5) and (15) we find

$$
J\left[m_{k}\right]=\frac{1}{8 \pi} \int_{\Sigma}\left(-\frac{1}{3} \mathrm{Y}_{I k} \wedge F^{I}+\frac{2}{3} \mathrm{~d}\left[g_{I J} \star F^{J} \Phi_{k}^{I}\right]\right)=\frac{\pi}{6} \int_{\mathcal{B}} \eta_{i j} \mathrm{~d} \mu_{I i k} \wedge \mathrm{d} \Phi_{j}^{I}
$$

where we have eliminated the total derivative term in the first integral by using the fact that $\Phi_{i}^{I}$ vanish at infinity. We can express this as an integral over $\partial \mathcal{B}$ (note that we can choose the integration constants appropriately to ensure $\mu_{I 1 k}$ and $\mu_{I 2 k}$ vanish on $I_{+}$and $I_{-}$respectively) and a similar computation that led to (18) leads to

$$
J\left[m_{k}\right]=\frac{\pi}{3} \sum_{[C]} \mu_{I k}[C] q^{I}[C]
$$

where $\mu_{I k}^{I}[C] \equiv-v_{a}^{i} \mu_{I i k}$ and $v_{a}^{i}$ are the set of integers characterizing the rod $I_{a}$ corresponding to the 2-cycle $C \in H_{2}(\Sigma)$. This shows how the source of the angular momenta in a soliton spacetime are the magnetic fluxes $q^{I}[C]$, weighted by the $\mu_{I k}[C]$.

Finally, for the electric charge, we have, using (5) and (15)

$$
Q_{I}=-\frac{1}{32 \pi} \int_{\Sigma} C_{I J K} F^{J} \wedge F^{K}=\frac{\pi}{8} \int_{\mathcal{B}} \eta_{i j} C_{I J K} \mathrm{~d} \Phi_{i}^{J} \wedge \mathrm{d} \Phi_{j}^{K}
$$

which leads to

$$
Q_{I}=-\frac{\pi}{4} \sum_{[C]} C_{I J K} \Phi^{J}[C] q^{K}[C]
$$

which expresses the total electric charge as a sum over the magnetic fluxes from each 2-cycle, weighted by the associated electric potentials $\Phi^{I}[C]$. 


\subsection{Black Holes Spacetimes Containing Solitons}

We now assume the spacetime contains a single connected black hole horizon. We will of course find the usual horizon terms in the first law, but also additional terms arising from the presence of 2-cycles in the exterior. We will also find terms associated to certain disc-topology surfaces which arise in black hole spacetimes with finite rods in their orbit space. Note that this possibility can occur even when there are no 2-cycles in the spacetime (e.g., for black rings and black lenses).

In the orbit space framework, a horizon $H$ is a finite rod $I_{H}$ upon which the Killing field $\xi$ goes null while the matrix $g\left(m_{i}, m_{j}\right)$ has rank 2 . On a Killing horizon, $R_{a b} \xi^{a} \xi^{b}=0$ and by standard arguments one can show that $i_{\xi} F^{I} \propto \xi, i_{m_{i}} i_{\xi} G^{I} \propto \xi$ on $H$. Using this we see the potentials $\Phi^{I}$ and $U_{I i}$ are all constant on this interval. We exploit this fact by 'integrating by parts' in the opposite way to rewrite the expression (17) as

$$
\int_{\mathcal{B}} \eta_{i j} \mathrm{~d} U_{I j} \wedge \mathrm{d} \Phi_{i}^{I}=-\int_{\partial \mathcal{B}} \eta_{i j} \Phi_{i}^{I} \mathrm{~d} U_{I j}
$$

where as before, there are no contributions from $\partial \mathcal{B}_{\infty}$ or $I_{ \pm}$. There is no contribution from $I_{H}$ either as the $U_{I i}$ are constant there. However, the presence of a horizon still manifests itself as follows. Suppose there is some finite rod $I_{D}$ upon which $\hat{m}_{1}=v_{D}^{i} m_{i}$ vanishes that lies adjacent to $I_{H}$ (i.e., it shares one endpoint $p \in \partial \mathcal{B}$ with $I_{H}$ ). Then $\hat{m}_{2}$ vanishes at the other endpoint of $I_{D}$ but not at $p$. Hence $I_{D}$ has the topology of a disc $D$ or 'cigar' with a boundary $S^{1}$ that intersecting the horizon $H$. There can be at most two such discs in a spacetime, corresponding to such intervals lying 'on either side' of $I_{H}$.

On such a disc define the flux charge

$$
\mathcal{Q}_{I}[D] \equiv \frac{1}{4} \int_{D}\left(\Theta_{I}+\frac{1}{2} C_{I J K} F^{J} \Phi_{H}^{K}\right)
$$

and note that the integrand is a closed 2-form, so that one can use any surface homologous to $D$ (with the same boundary) to evaluate the integral. We find

$$
\frac{\pi}{2} \int_{I_{D}} \eta_{i j} \Phi_{i}^{I} \mathrm{~d} U_{I j}=\frac{\pi}{2} \hat{\Phi}_{1}^{I} \int_{I_{D}} \mathrm{~d} \hat{U}_{I 2}=\Phi^{I}[D] \mathcal{Q}_{I}[D]
$$

and $\Phi^{I}[D] \equiv v_{D}^{i} \Phi_{i}^{I}$. Similarly, starting from the mass variation formula in the presence of a horizon,

$$
\frac{1}{8 \pi} \int_{\Sigma} \Theta_{I} \wedge \delta F^{I}+\frac{1}{8 \pi} \int_{H} \Phi^{I} \delta\left(g_{I J} \star F^{J}\right)=\Phi_{H}^{I} \delta Q_{I}+\frac{\pi}{2} \int_{B} \eta_{i j} \mathrm{~d} U_{I j} \wedge \mathrm{d} \delta \Phi_{i}^{I} .
$$

We express the final integral on the right hand side as

$$
\int_{\mathcal{B}} \eta_{i j} \mathrm{~d} U_{I j} \wedge \mathrm{d} \delta \Phi_{i}^{I}=-\int_{\partial \mathcal{B}} \eta_{i j} \delta \Phi_{i}^{I} \mathrm{~d} U_{I j}
$$

Once again using the fact $U_{I i}$ is constant on $I_{H}$, the only contributions will come from disc rods $I_{D}$ and finite rods $I_{a}$ corresponding to 2-cycles $C \in H_{2}(\Sigma)$. In particular,

$$
\int_{I_{D}} \eta_{i j} \delta \Phi_{i}^{I} \mathrm{~d} U_{I j}=-\frac{2}{\pi} \delta \Phi^{I}[D] \mathcal{Q}_{I}[D],
$$

where we have made use of the fact $v_{D}^{i} \delta \Phi_{i}^{I}$ is constant on the $I_{D}$. There are analogous terms over the finite rods, with analogous definitions for $\Phi^{I}[C]$ and $\mathcal{Q}[C]$. Collecting these results we arrive at the following main result [10]: 
Theorem 1. Consider an asymptotically flat $\mathbb{R} \times U(1)^{2}$-invariant solution $\left(g, F^{I}, \chi^{A}\right)$ of the theory (3) with a domain of outer communication $\mathbb{R} \times \Sigma$. Then the mass formula

$$
M=\frac{3 \kappa A_{H}}{16 \pi}+\frac{3}{2} \Omega_{i} J_{i}+\Phi_{H}^{I} Q_{I}+\frac{1}{2} \sum_{[C]} \mathcal{Q}_{I}[C] \Phi^{I}[C]+\frac{1}{2} \sum_{[D]} \mathcal{Q}_{I}[D] \Phi^{I}[D]
$$

holds, where $[C]$ is a basis for $\mathrm{H}_{2}[\Sigma]$ and $[D]$ are disc topology surfaces ending on the horizon. Furthermore, consider $\mathbb{R} \times U(1)^{2}$-invariant variations $\left(\delta g, \delta F^{I}, \delta \chi^{A}\right)$ satisfying the linearized field equations. Then the variation of the mass is given by

$$
\delta M=\frac{\kappa \delta A_{H}}{8 \pi}+\Omega_{i} \delta J_{i}+\Phi_{H}^{I} \delta Q_{I}+\sum_{[C]} \mathcal{Q}_{I}[C] \delta \Phi^{I}[C]+\sum_{[D]} \mathcal{Q}_{I}[D] \delta \Phi^{I}[D]
$$

Some remarks are in order. Firstly, if there are no horizons, then the usual black hole terms in (34) and (35) vanish, as well as terms involving discs. We then obtain different, but equivalent, expressions for the mass and mass variation formulae for solitons given in (21) and (22). Secondly, if there are no 2-cycles (solitons) in the spacetime, then we recover the standard laws of black hole mechanics, with the additional advantage that we can now take into account non-spherical horizon topologies via the disc terms. Indeed, additional terms in the Smarr relation and first law for black rings were originally observed by Emparan [18] and later given a derivation by Copsey and Horowitz [19]. In contrast here the extra terms arise in a natural way as a consequence of the structure of the orbit space, as has recently been shown in [20]. Finally, it is interesting that variations of intensive variables (e.g., $\delta \Phi_{i}^{I}[D]$ ) appear in the first law, rather than those of extensive quantities. The significance of this remains to be explored. but at least for black rings and black lenses, the quantities $\Phi_{i}^{I}[D]$ and $\Phi_{i}^{I}[C]$ capture the notion of the 'dipole charges' that characterize these spacetimes [8].

We conclude by noting that additional terms to the standard first law can arise in other settings than the ones considered here; for example, if we consider non-asymptotically flat spacetimes that admit translational isometries, such as $p$-brane supergravity solutions, there are additional 'tension' terms associated to variations of the compact directions [21]. In addition, one can have dipole terms arising if there is a canonical $S^{2}$ at spatial infinity (see, e.g., [22]) and spacetimes admitting Kaluza-Klein bubbles [23].

\subsection{A Gravitational 1-Soliton Spacetime $\mathcal{M}=\mathbb{R}^{4} \# \mathbb{C} \mathbb{P}^{2}$}

Here we will consider an explicit example in the simple five-dimensional minimal supergravity theory. The solution describes a simple non-supersymmetric asymptotically flat soliton on $\mathbb{C P}^{2}$ with a point removed [17], and is sufficiently complicated to exhibit the important features discussed above. The metric and Maxwell field are given by

$$
\begin{aligned}
\mathrm{d} s^{2} & =-\frac{r^{2} W(r)}{4 b(r)^{2}} \mathrm{~d} t^{2}+\frac{\mathrm{d} r^{2}}{W(r)}+\frac{r^{2}}{4}\left(\sigma_{1}^{2}+\sigma_{2}^{2}\right)+b(r)^{2}\left(\sigma_{3}+f(r) \mathrm{d} t\right)^{2} \\
F & =\frac{\sqrt{3 q}}{2} \mathrm{~d}\left[\left(\frac{1}{r^{2}}\right)\left(\frac{j}{2} \sigma_{3}-\mathrm{d} t\right)\right]
\end{aligned}
$$

where $\sigma_{i}$ are the left-invariant one-forms on $\operatorname{SU}(2)$ and $\psi \sim \psi+4 \pi, \phi \sim \phi+2 \pi$, and $\theta \in(0, \pi)$ to ensure asymptotic flatness of $g$ as $r \rightarrow \infty$. The functions appearing above are

$$
\begin{aligned}
W(r) & =1-\frac{2}{r^{2}}(p-q)+\frac{q^{2}+2 p j^{2}}{r^{4}} \quad f(r)=-\frac{j}{2 b(r)^{2}}\left(\frac{2 p-q}{r^{2}}-\frac{q^{2}}{r^{4}}\right) \\
b(r)^{2} & =\frac{r^{2}}{4}\left(1-\frac{j^{2} q^{2}}{r^{6}}+\frac{2 j^{2} p}{r^{4}}\right)
\end{aligned}
$$


where $p, q, j \in \mathbb{R}$. The set $(g, F)$ are a solution to minimal supergravity, obtained by setting $N=1, g_{I J}=2$, and $C_{I J K}=16 / \sqrt{3}$ and all scalars to be constant in (3). If one takes

$$
p=\frac{r_{0}^{4}\left(r_{0}^{2}-j^{2}\right)}{2 j^{4}} \quad q=-\frac{r_{0}^{4}}{j^{2}}
$$

then it can be shown that the geometry is complete and $r \geq r_{0}$. The Killing field $\partial_{\psi}$ degenerates at $r=r_{0}$ and the manifold near that point is diffeormorphic to $\mathbb{R}_{t} \times S^{2} \times\{p t\}$ provided there is no conical singularity at $r=r_{0}$, which requires [20]

$$
\left(1-\frac{r_{0}^{2}}{j^{2}}\right)\left(2+\frac{r_{0}^{2}}{j^{2}}\right)^{2}=1
$$

which fixes $r_{0}^{2} / j^{2} \approx 0.870385$, and in particular $r_{0}^{2}<j^{2}$. One can check that $g^{t t}, g_{t t}<0$ everywhere, so the spacetime is stably causal with no ergoregions and the $t=t_{0}$ surfaces are Cauchy hypersurfaces. In summary we have a one-parameter family of smooth, horizonless asymptotically flat stationary soliton spacetimes with $\mathbb{R} \times S U(2) \times U(1)$ symmetry.

The spacetime has a single 2-cycle at $r=r_{0}$ with associated magnetic flux [20]

$$
q[C]=\frac{1}{4 \pi} \int_{S^{2}} F=\frac{\sqrt{3} r_{0}^{2}}{4 j}
$$

and the various potential functions can be worked out after some computation (we choose our basis of the $U(1)^{2}$ generators with $2 \pi$ periodic orbits to be $\partial_{\hat{\psi}} \equiv 2 \partial_{\psi}$ and $\partial_{\phi}$ ):

$$
\Phi_{\xi}=\frac{\sqrt{3} q}{2 r^{2}}, \quad \Phi_{\hat{\psi}}=-\frac{\sqrt{3} q j}{2 r^{2}}, \quad \Phi_{\phi}=-\frac{\sqrt{3} q j \cos \theta}{4 r^{2}} .
$$

and

$$
U_{\hat{\psi}}=\frac{\sqrt{3} j q}{r^{2}}\left(\frac{q}{r^{2}}-1\right), \quad U_{\phi}=\frac{\sqrt{3} j q \cos \theta}{2 r^{2}}\left(\frac{q}{r^{2}}-1\right) .
$$

Finally, we have

$$
\begin{gathered}
\mu_{\psi \psi}=-\frac{\sqrt{3} q^{2} j^{2}}{4 r^{4}}+\frac{\sqrt{3} q}{4}, \quad \mu_{\phi \psi}=\frac{\sqrt{3} q \cos \theta}{4}\left(1-\frac{q j^{2}}{r^{4}}\right) \\
\mu_{\phi \phi}=-\frac{\sqrt{3} q^{2} j^{2} \cos ^{2} \theta}{4 r^{4}}-\frac{\sqrt{3} q}{4}, \quad \mu_{\psi \phi}=-\frac{\sqrt{3} q \cos \theta}{4}\left(1+\frac{q j^{2}}{r^{4}}\right)
\end{gathered}
$$

where integration constants are chosen appropriately using the prescription given above. Using the above formula one finds

$$
\frac{\Psi[C] q[C]}{2}=\frac{3 \pi}{8}\left(\frac{r_{0}}{j}\right)^{4}\left(j^{2}+r_{0}^{2}\right)
$$

which is indeed the ADM mass $M$ of the spacetime in accordance with (21). Furthermore,

$$
Q=-\frac{4 \pi}{\sqrt{3}} \Phi[C] q[C]=-\frac{\sqrt{3} \pi r_{0}^{4}}{2 j^{2}}
$$

and since the 2-cycle is specified by the vanishing of $\partial_{\hat{\psi}}$, using the formula (25) we find

$$
J_{\psi}=\frac{\pi r_{0}^{6}}{4 j^{3}}, \quad J_{\phi}=0
$$


where in the second equality we observe that $\chi_{\psi \phi}=0$ on $C$ using (40). These expressions agree with the expressions for $Q, J_{i}$ obtained using the standard definitions. It is important to note that the first law for soliton mechanics (22) reads $\delta M=\Psi[C] \delta q[C]$ in this example. A calculation reveals [20]

$$
\mathrm{d} M-\Psi[C] \mathrm{d} q[C]=\frac{3 \pi r_{0}^{5}}{4 j^{5}}\left(j \mathrm{~d} r_{0}-r_{0} \mathrm{~d} j\right)
$$

Thus the relation (22) holds provided the regularity condition (41) holds, i.e., $r_{0}^{2} / j^{2}$ is constant (although the precise value of the constant does not actually appear). This indicates the key role that regularity of the spacetime plays in the first law. This is in contrast to the Smarr type relation (46) for the mass, which holds without explicit use of the regularity condition.

\section{Discussion}

The moduli space of stationary, $U(1)^{2}$-invariant black hole solutions in five dimensions clearly has a rich structure that remains to be fully explored. It is natural to interpret (35) as an equation for the tangent space at a given point in the solution space. The orbit space framework provides a framework to characterize these solutions, although at present it does not address the issue of existence, which is typically much more difficult than the corresponding uniqueness problem. In the usual $D=4$ Einstein-Maxwell system, solutions are specified by three conserved charges $(M, Q, J)$; in $D=5$ one must also include the spacetimes's rod data. These in turn give rise to various fluxes $q^{I}[C], \mathcal{Q}^{I}[C]$ through 2-cycles $[C]$ and corresponding quantities through disc topology surfaces $[D]$ attached to the horizon. There are few examples of solutions that describe an asymptotically flat spacetime containing a black hole-soliton configuration-for example, see [16]. The construction of this solution, however, depends crucially on supersymmetry. In this case, one can show that the additional "interaction" terms in (35) and (34) vanish. This is similar to the fact that the surface gravity $\kappa$, angular velocities $\Omega_{i}$, and electric potentials $\Phi_{H}^{I}$ vanish on the horizon of a supersymmetric black hole.

We emphasize though that in the first law (35) it is the variations of the potentials, $\delta \Phi^{I}[C], \delta \Phi^{I}[D]$ that appear, rather than e.g., $\delta q^{I}[C], \delta q^{I}[D]$. In contrast, for pure soliton spacetimes, the mass-variation formula (22) and Smarr-type formulae (22) and (21) take a standard form, although of course there is no thermodynamic interpretation in this case. Understanding the role the fluxes and potentials play will be necessary to understand black hole thermodynamics in five dimensions. In particular, the extra terms characterize the interaction between the horizon and the presence of solitons in the domain of outer communication. Such terms may be relevant when considering the possibility that a black hole can "shed" a soliton. More broadly, it would be interesting to see if there is a connection between this work and the general dynamical stability problem for black holes.

A natural generalization to pursue would be to consider black hole-solition spacetimes that are asymptotically globally Anti-de Sitter. The definition of the Komar mass used to derive the Smarr relation is divergent in this case (there is a divergent term in the volume integral appearing on the right hand side of (9)). Therefore a different approach (e.g., using counterterms) than the ones used in this paper would be required, using an appropriately well-defined mass. To derive a general first law in this setting it may be useful to study explicit examples of gravitational solitons in $\mathrm{AdS}_{5}$ [24] and their physical properties [25]. Extending beyond the case of globally $\mathrm{AdS}_{5}$ asymptotics, one can also consider the thermodynamics of black string spacetimes with a negative cosmological constant with compact spatial directions in the asymptotic region [26].

Acknowledgments: This research is supported by a National Science and Engineering and Research Council of Canada Discovery Grant. I would like to especially thank my collaborator James Lucietti.

Conflicts of Interest: The author declares no conflict of interest. 


\section{References}

1. Chrusciel, P.T.; Lopes Costa, J.; Heusler, M. Stationary Black Holes: Uniqueness and Beyond. Living Rev. Relativ. 2012, 15, 7 .

2. Bardeen, J.M.; Carter, B.; Hawking, S.W. The Four laws of black hole mechanics. Commun. Math. Phys. 1973, 31, 161-170.

3. Emparan, R.; Reall, H.S. Black Holes in Higher Dimensions. Living Rev. Relativ. 2008, 11, 6.

4. Hollands, S.; Ishibashi, A. Black hole uniqueness theorems in higher dimensional spacetimes. Class. Quantum Gravity 2012, 29, 163001.

5. Myers, R.C.; Perry, M.J. Black Holes in Higher Dimensional Space-Times. Ann. Phys. 1986, 172, $304-347$.

6. Emparan, R.; Reall, H.S. A rotating black ring solution in five-dimensions. Phys. Rev. Lett. 2002, 88, 101101.

7. Kunduri, H.K.; Lucietti, J. Supersymmetric Black Holes with Lens-Space Topology. Phys. Rev. Lett. 2014, 113, 211101.

8. Kunduri, H.K.; Lucietti, J. Black lenses in string theory. Phys. Rev. D 2016, 94, 064007.

9. Hollands, S.; Yazadjiev, S. Uniqueness theorem for 5-dimensional black holes with two axial Killing fields. Commun. Math. Phys. 2008, 283, 749-768.

10. Kunduri, H.K.; Lucietti, J. The first law of soliton and black hole mechanics in five dimensions. Class. Quantum Gravity 2014, 31, 032001.

11. Nedkova, P.G.; Yazadjiev, S.S. On the Thermodynamics of 5D Black Holes on ALF Gravitational Instantons. Phys. Rev. D 2011, 84, 124040.

12. Gibbons, G.W. Supergravity vacua and solitons. In Duality and Supersymmetric Theories; Cambridge University Press: Cambridge, UK, 1997.

13. Friedman, J.L.; Schleich, K.; Witt, D.M. Topological censorship. Phys. Rev. Lett. 1993, 71, 1486.

14. Ashtekar, A.; Corichi, A.; Sudarsky, D. Hairy black holes, horizon mass and solitons. Class. Quantum Gravity 2001, 18, 919-940.

15. Bena, I.; Warner, N.P. Black holes, black rings and their microstates. In Supersymmetric Mechanics—Vol. 3; Springer: Berlin/Heidelberg, Germany, 2008.

16. Kunduri, H.K.; Lucietti, J. Black hole non-uniqueness via spacetime topology in five dimensions. J. High Energy Phys. 2014, 2014, 82.

17. Gibbons, G.W.; Warner, N.P. Global structure of five-dimensional fuzzballs. Class. Quantum Gravity 2014, 31, 025016.

18. Emparan, R. Rotating circular strings, and infinite nonuniqueness of black rings. J. High Energy Phys. 2004, $2004,064$.

19. Copsey, K.; Horowitz, G.T. The Role of dipole charges in black hole thermodynamics. Phys. Rev. D 2006, $73,024015$.

20. Gunasekaran, S.; Hussain, U.; Kunduri, H.K. Soliton mechanics. Phys. Rev. D 2016, 94, 124029.

21. Townsend, P.K.; Zamaklar, M. The first law of black brane mechanics. Class. Quantum Gravity 2001, 18, 5269-5286.

22. Stelea, C.; Schleich, K.; Witt, D. On squashed black holes in Godel universes. Phys. Rev. D 2008, 78, 124006.

23. Yazadjiev, S.S.; Nedkova, P.G. Magnetized configurations with black holes and Kaluza-Klein bubbles: Smarr-like relations and first law. Phys. Rev. D 2009, 80, 024005.

24. Ross, S.F. Non-supersymmetric asymptotically $\mathrm{AdS}_{5} \times S^{5}$ smooth geometries. J. High Energy Phys. 2006, 2006, doi:10.1088/1126-6708/2006/01/130.

25. Compere, G.; Copsey, K.; de Buyl, S.; Mann, R.B. Solitons in Five Dimensional Minimal Supergravity: Local Charge, Exotic Ergoregions, and Violations of the BPS Bound. J. High Energy Phys. 2009, $2009,047$.

26. Brihaye, Y.; Radu, E.; Stelea, C. Black strings with negative cosmological constant: Inclusion of electric charge and rotation. Class. Quantum Gravity 2007, 24, 4839-4870.

(C) 2017 by the author; licensee MDPI, Basel, Switzerland. This article is an open access article distributed under the terms and conditions of the Creative Commons Attribution (CC-BY) license (http://creativecommons.org/licenses/by/4.0/). 